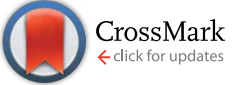

Cite this: RSC Adv., 2017, 7, 17368
Received 17th January 2017 Accepted 8th March 2017

DOI: $10.1039 / c 7 r a 00705 a$

rsc.li/rsc-advances

\section{Theoretical investigation on the effect of ancillary ligand modification for highly efficient phosphorescent platinum(II) complex design $\uparrow$}

\author{
Hong-Wei Fan, Fu-Quan Bai, ${ }^{\star}$ Zhi-Xiang Zhang, Yu Wang, Ze-Xing Qu, \\ Rong-Lin Zhong and Hong-Xing Zhang*
}

In this study, density functional theory (DFT) and time-dependent density functional theory (TDDFT) calculations were employed to investigate the geometries, electronic structures, reorganization energy ( $\lambda$ ) and photophysical properties of four cyclometalated Pt(॥) complexes (bzq)Pt(dpm) (1), (bzq)Pt(ppy) (2), (bzq)Pt(Ncaz) (3) and (bzq)Pt(Ndbt) (4) (where bzq = benzo[h]quinoline, dpm = dipivolylmethanoate, ppy $=2$-phenylpyridine, Ncaz $=N$-substituted carbazole and Ndbt $=N$-substituted dibenzothiophene). In addition, the radiative decay processes and zero-field splitting were calculated based on the spin-orbit coupling (SOC) effect, and nonradiative decay pathways were discussed to evaluate the phosphorescence efficiency qualitatively. All the complexes retain the bzq as a cyclometalated ligand and our research focuses on the role recognition of another ancillary ligand modification theoretically. According to the results, in complexes 2-4 replacing the dpm with different ligands shows better rigidity which may weaken the nonradiative decay pathways and enhance the capability of charge transfer. Furthermore, complexes 1-4 tend to be bluish-green luminescent materials, and the emission wavelengths of 1,2 and 4 are close to each other due to the similar excited state energy levels and electronic density distribution. Compared with complex 1 , the radiative decay rate constants $\left(k_{r}\right)$ of $2-4$ are greatly increased. Therefore, the designed complexes would be potential phosphorescence materials because of their high phosphorescence quantum efficiency and complex 3 can also serve as a promising bipolar transporting material due to its better charge transfer balance character.

\section{Introduction}

Over the past decades, organic light emitting diodes (OLEDs) have obtained a great deal of attention due to their flexibility in flat-panel displays. ${ }^{1,2}$ They are widely used in digital products and solid-state lighting devices..$^{3-6}$ Phosphorescent transitionmetal compounds have excellent performances as triplet dopant emitters in OLED emission layers, especially those of Ir(iii) and Pt(II) complexes. They are heavy metal atoms that can induce strong spin-orbit coupling (SOC) and promote an effective intersystem crossing (ISC), which results in higher quantum efficiency. ${ }^{7-11}$ Platinum which has the second largest SOC constant, tends to form planar structure due to the $\mathrm{d}^{8}$ electron configuration of the $\mathrm{Pt}(\mathrm{II})$ ion. The ligand of cyclometalated $\mathrm{Pt}(\mathrm{II})$ complexes can be designed as bidentate,

International Joint Research Laboratory of Nano-Micro Architecture Chemistry, Laboratory of Theoretical and Computational Chemistry, Institute of Theoretical Chemistry, Jilin University, Changchun 130023, China. E-mail: baifq@jlu.edu.cn; zhanghx@mail.jlu.edu.cn

$\dagger$ Electronic supplementary information (ESI) available: Details of calculated molecular orbital (MO) compositions, NTOs, the coupling energy levels and transition characters analysis between singlet excited states and triplet excited states for complexes 1-4. See DOI: 10.1039/c7ra00705a tridentate and tetradentate. The diversity of these ligands can greatly affect the phosphorescence properties. ${ }^{12-16}$ As we know, the singlet excited states convert to the triplet states through ISC due to the SOC and then the triplet excited states return to the ground state, phosphorescence can be achieved through the radiative decay process. Nevertheless, if nonradiative decay holds a large proportion in the whole decay process, there will be weak or no phosphorescence. ${ }^{17}$ Therefore, the key to improve the efficiency of electroluminescent emitters are to increase the radiative decay rate constants and decrease the nonradiative decay rate constants. ${ }^{18}$

Generally, chelating ligands of transition-metal complexes, which play a critical role in the OLEDs, include the cyclometalated ligands and ancillary ligands. The cyclometalated ligands have influence on the charge transition between metal and ligand (MLCT) at the excited states, and then alter the emission wavelengths. ${ }^{19}$ Moreover, ancillary ligands provide the ligand fields and affect the relative energy of metal center, such as $\beta$-diketonate and picolinate. ${ }^{20,21}$ Previous researches have demonstrated that the phosphorescence properties would be easily tuned mostly by the variation of cyclometalated ligands, while the change of ancillary ligands has little influence..$^{22,23}$ Recently, ancillary ligands can affect the phosphorescence 
efficiency evidently through altering the properties of excitedstate was recorded. ${ }^{24}$ Thus, the modification of ancillary ligands becomes the focus for the highly efficient of transitionmetal complexes. ${ }^{25,26}$ In this respect, the heteroleptic $\mathrm{C}^{\wedge} \mathrm{N}$ ligands such as phenyl-pyrazole (ppy) and phenyl-pyrazole (ppz) were widely applied in transition metal complexes. ${ }^{27-30}$ The $\mathrm{C}^{\wedge} \mathrm{N}$ ligand can bind to metals forming a five-membered chelate through the $\mathrm{N}$ atom of pyridine ring and $\mathrm{C}$ atom of phenyl. The anionic $\mathrm{C}$ atom related to the metal centre is a strong $\sigma$-donor while the pyridyl ring maintains a favourable $\pi$-acceptor. The $\mathrm{C}^{\wedge} \mathrm{N}$ ligands provide the metal ion a strong ligand field which leads to a higher energy of deactivating $\mathrm{d}-\mathrm{d}$ states and reduces the thermal quenching compared to homologous $\mathrm{N}^{\wedge} \mathrm{N}$ ligands..$^{31,32}$ Lately, the carbazole group has been used to improve the rigidity and performance of complexes. As reported by Hang and co-workers, a series of highly efficient cyclometalated $\mathrm{Pt}(\mathrm{II})$ complexes were synthesized and the maximum external quantum efficiency value as high as $23.7 \%$ were achieved by introducing a carbazole group in ligand. ${ }^{33}$ Based on this knowledge, the $\mathrm{N}$ atom on the carbazole group is substituted by the $\mathrm{S}$ atom to foresee new structure. Recent research in Thompson group, they have synthesized and reported a series of novel bidentate $\mathrm{Pt}(\mathrm{II})$ complexes. ${ }^{34}$ Among these complexes, the suitable complex $\mathbf{1}$ has still the small value of nonradiative decay rate constant. Based on the complex $\mathbf{1}$, complexes 2-4 in Fig. 1 are tried to be designed by replacing the $\mathrm{dpm}$ ligand with different ancillary ligands mentioned above to seek the better candidates. Herein, as the highly efficient phosphorescent OLED materials, the newly designed complexes are expected to improve the radiative decay rate constant and strengthen the stability.

In order to gain a deep insight into the effects of ancillary ligands on the photophysical properties and quantum efficiency of these complexes, DFT and TDDFT methods have been adopted to investigate the electronic structures, charge transport abilities and emission properties of the complexes 1-4. The radiative decay rate $\left(k_{\mathrm{r}}\right)$ and zero-filed splitting values are quantitatively evaluated by the calculation of SOC matrix elements; nonradiative decay rate $\left(k_{\mathrm{nr}}\right)$ is qualitatively discussed via energy gap law and the relative energy between metalcentered $\left({ }^{3} \mathrm{MC}\right)$ state and minimum energy crossing point (MECP). ${ }^{17,35,36}$ Reorganization energy $(\lambda)$ is an important factor

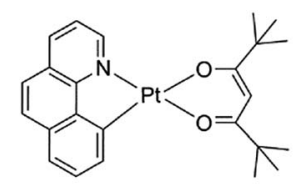

1:(bzq)Pt(dpm)

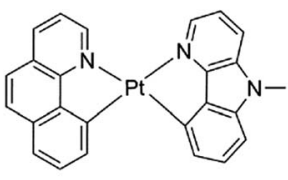

3:(bzq)Pt(Ncaz)

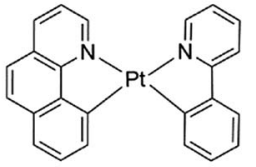

2:(bzq)Pt(ppy)

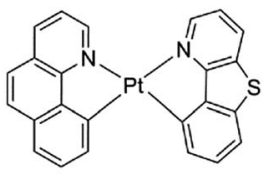

4:(bzq)Pt(Ndbt) in evaluating the balance between the electron and hole transport of the luminescent materials. The theoretical calculation results reveal that complexes 2 and $\mathbf{4}$ would be better phosphorescent materials for the high phosphorescence quantum efficiency and complex 3 can serve as promising bipolar candidate due to the balanced charge carrier injection and transport feature. We hope that our preliminary study could provide useful information for the further design and application of highly efficient luminescent material in OLEDs.

\section{Theory of computation}

\subsection{Theoretical background}

Generally speaking, the phosphorescence quantum efficiency of transition metals is decided by radiative decay and nonradiative decay rates. According to the energy gap law, the nonradiative decay rate constant $\left(k_{\mathrm{nr}}\right)$ from $\mathrm{T}_{m}$ to $\mathrm{S}_{0}$ states can be evaluated by the equation: $:^{37,38}$

$$
k_{\mathrm{nr}}\left(\mathrm{T}_{m} \rightarrow \mathrm{S}_{0}\right) \propto \exp \left\{-\beta\left[E\left(\mathrm{~T}_{m}\right)-E\left(\mathrm{~S}_{0}\right)\right]\right\}
$$

The parameter $\beta$ is involved with the structural distortion between the ground and correlative excited triplet states. $\left[E\left(\mathrm{~T}_{m}\right)\right.$ $\left.-E\left(\mathrm{~S}_{0}\right)\right]$ is the energy gap between excited state and ground state. Hence, the smaller structural distortion and larger energy gap between excited triplet and ground state would be beneficial to decrease the nonradiative decay rate.

On the basis of Born-Oppenheimer approximation and the first order perturbation theory, the phosphorescent radiative decay rate constant $\left(k_{\mathrm{r}}\right)$ that from $\mathrm{T}_{m}$ to $\mathrm{S}_{0}$ can be expressed as follows: ${ }^{39,40}$

$k_{\mathrm{r}}{ }^{\alpha}\left(\mathrm{T}_{m} \rightarrow \mathrm{S}_{0}\right)=\frac{16 \times 10^{6} \pi^{3} E\left(\mathrm{~T}_{m}\right)^{3} \eta^{3}}{3 h \varepsilon_{0}}\left\{\sum_{n} \frac{\left\langle\mathrm{T}_{m}{ }^{\alpha}\left|H_{\mathrm{SOC}}\right| \mathrm{S}_{n}\right\rangle}{E\left(\mathrm{~S}_{n}\right)-E\left(\mathrm{~T}_{m}\right)} \mathrm{M}_{\mathrm{S}_{n}}\right\}^{2}$

where $\eta$ means the refractive index of the cyclohexane solution which is taken to be 1.43. $E\left(\mathrm{~S}_{n}\right)$ represents the vertical excitation energy from the $n$th singlet excited $\left(\mathrm{S}_{n}\right)$ to ground state $\left(\mathrm{S}_{0}\right)$ transition. $E\left(\mathrm{~T}_{m}\right)$ represents the vertical excitation energy from the $m$ th triplet excited state ( $\left.\mathrm{T}_{m}\right)$ to $\mathrm{S}_{0}$ transition. $\left\langle\mathrm{T}_{m}{ }^{\alpha}\left|H_{\mathrm{SOC}}\right| \mathrm{S}_{n}\right\rangle$ is the SOC matrix element $(\alpha=x, y$ or $z) . M_{\mathrm{S}_{n}}$ is the transition dipole moment from $S_{0}$ to $S_{n}$, which can be defined by the following equation:

$$
M_{\mathrm{S}_{n}}=\left(\frac{3 h e^{2}}{8 \pi^{2} m_{\mathrm{e}} c} \times \frac{f_{n}}{E\left(\mathrm{~S}_{n}\right)}\right)^{\frac{1}{2}}
$$

It is obvious that the $M_{\mathrm{S}_{n}}$ is connected with the oscillator strength $\left(f_{n}\right)$ and transition energy $\left(E\left(\mathrm{~S}_{n}\right)\right)$. Where $e$ is the electron charge; $m_{\mathrm{e}}$ and $c$ are the mass of an electron and light speed. Combined with the eqn (2) and (3), the $k_{\mathrm{r}}$ is represented in the form:

$$
k_{\mathrm{r}}^{\alpha}\left(\mathrm{T}_{m} \rightarrow \mathrm{S}_{0}\right)=\frac{\eta^{3}}{1.5} E\left(\mathrm{~T}_{m}\right)^{3}\left\{\sum_{n} \frac{\left\langle\mathrm{T}_{m}{ }^{\alpha}\left|H_{\mathrm{SOC}}\right| S_{n}\right\rangle}{E\left(\mathrm{~S}_{n}\right)-E\left(\mathrm{~T}_{m}\right)}\left(\frac{f_{n}}{E\left(\mathrm{~S}_{n}\right)}\right)^{1 / 2}\right\}^{2}
$$

Fig. 1 Chemical structures of the investigated complexes 1-4. 
Under the Boltzmann statistics distribution, the triplet sublevels are postulated at thermal equilibrium. Thus, the $k_{\mathrm{r}}$ can be calculated as the vectorial summation of $k_{\mathrm{r}}{ }^{\alpha}$ values as follows: $:^{41,42}$

$$
k_{\mathrm{r}}=\frac{1}{3} \sum_{\alpha} k_{\mathrm{r}}{ }^{\alpha}
$$

As we all know, the triplet state splits into three spin triplet sublevels duo to the SOC effect. Zero-field splitting (ZFS) is defined as the energy splitting between the first and third sublevel, which is related to the composition of MLCT in emission excited and the magnitude of $k_{\mathrm{r}}$ values. $^{\mathbf{4 3 , 4 4}}$ The $\Delta E(\mathrm{ZFS})$ can be described in the form:

$$
\Delta E\left(\mathrm{~T}_{m}{ }^{\alpha}\right)=\sum_{n} \frac{\left|\left\langle\mathrm{T}_{m}{ }^{\alpha}\left|H_{\mathrm{SOC}}\right| \mathrm{S}_{n}\right\rangle\right|}{E\left(\mathrm{~S}_{n}\right)-E\left(\mathrm{~T}_{m}\right)}
$$

where $\alpha$ is the three sublevels of energy shift.

\subsection{Computational details}

All the calculations were performed by the Gaussian 09 software package with a tight self-consistent convergence threshold to ensure optimization and wave functions convergence. ${ }^{45}$ The geometry optimization and phosphorescent properties were evaluated by DFT and TDDFT. In the process of optimization, vibrational frequencies were calculated with no imaginary frequencies, indicating the optimized structures are at the minima of the potential energy surface. Since the experimental data was obtained in cyclohexane solution, the solvent effect by utilizing the Polarizable Continuum Model (PCM) was taken into account in the geometry optimization and phosphorescence properties. ${ }^{\mathbf{4 6 , 4 7}}$ In addition, Hay and Wadt's ${ }^{\mathbf{4 8}}$ quasirelativistic effective core potential (ECP) with 18 valence electrons as well as the "double- $\zeta$ " quality basis set LANL2DZ were adopted for platinum atom and the 6-31G(d) basis set was applied to the non-metallic atoms such as $\mathrm{C}, \mathrm{H}, \mathrm{O}, \mathrm{N}$ and $\mathrm{S}$ atoms.

It is important to choose a reliable exchange-correlation functional for the current system. Considering the chargetransfer of ligand to metal in transition metal complexes, five widely used exchange-correlation functionals containing the B3LYP, ${ }^{49}$ B3P86, ${ }^{50}$ PBE0, ${ }^{51}$ M062X, ${ }^{52}$ and CAM-B3LYP ${ }^{53}$ were employed for the singlet ground state of complex 1 compared to its crystal X-ray structure. The corresponding parameters of the optimized ground state geometry together with experimental values are listed in Table 1 . The results show that PBE0 can give an accurate geometry structure among these functionals because its average relative error is the smallest. Thus, the optimization of the ground states and the lowest triplet excited states for complexes 1-4 were calculated with PBE0 functional in the studied system.

Owing to spin flipped in triplet states, we also find a responsible method to predict the emission properties, five different functionals were performed on the lowest-lying emission wavelength of triplet state for complex 1. On one hand, through the calculation of five functionals, the transition properties of lowest triplet excited state $\left(\mathrm{T}_{1}\right)$ are similar. On the other hand, the calculated lowest-lying emission wavelengths are $697 \mathrm{~nm}, 667 \mathrm{~nm}, 739 \mathrm{~nm}, 658 \mathrm{~nm}, 537 \mathrm{~nm}$ with PBE0, B3P86, CAM-B3LYP, B3LYP, M06-2X functionals, respectively. From the result, the wavelength calculated by M06-2X functional is in good agreement with the experimental value of $505 \mathrm{~nm}$. Besides, for the assessment of singlet-triplet transition energies based on the TDDFT, M06-2X is regarded as the most accurate functional which can adopt inner track to estimate with the mean absolute error close to $0.25 \mathrm{eV}$ in most cases. ${ }^{54} \mathrm{In}$ short, the PBE0 functional was used to optimize the singlet ground state and the triplet state geometric structures for all complexes in the current system, while the emission energies were analyzed by M06-2X functional.

\section{Results and discussion}

\subsection{Geometries in ground and lowest-lying triplet excited states}

The optimized structures at ground-state along with some atomic labels of the complexes are shown in Fig. 2 . It can be seen that these $\mathrm{Pt}$ (II) complexes with $\mathrm{d}^{8}$ configuration present a square geometry. To gain a better understanding of the influence about different ligands, some changes of geometry parameters for all complexes are discussed in both $S_{0}$ and $T_{1}$ states. The main bond distances and angles between metal center Pt(II) and relevant atoms as well as dihedral angle at $\mathrm{S}_{0}$ and $\mathrm{T}_{1}$ states are listed in Table 2.

From the results, the bond lengths of Pt-N1 are about 0.182 A longer than $\mathrm{Pt}-\mathrm{C} 2$ in complexes 1-4, indicating that the coordinated bond between atoms $\mathrm{C}$ and $\mathrm{Pt}$ is stronger than the

Table 1 Optimized geometric parameters at the singlet ground state of complex 1 by different functionals as well as the experimental values ( $\AA$ )

\begin{tabular}{llllllllllll}
\hline Item & Expt $^{a}$ & PBE0 & Relative error & B3LYP & Relative error & B3P86 & Relative error & M062X & Relative error & CAM- B3LYP & Relative error \\
\hline Pt-N1 & 1.983 & 2.013 & $1.5 \%$ & 2.039 & $2.8 \%$ & 2.016 & $1.7 \%$ & 2.039 & $2.8 \%$ & 2.030 \\
Pt-C2 & 1.990 & 1.978 & $0.6 \%$ & 1.996 & $0.3 \%$ & 1.978 & $0.6 \%$ & 1.967 & $1.2 \%$ & 1.988 \\
Pt-O3 & 2.029 & 2.015 & $0.7 \%$ & 2.034 & $0.3 \%$ & 2.014 & $0.7 \%$ & 2.037 & $0.4 \%$ & 2.018 \\
Pt-O4 & 2.032 & 2.110 & $3.8 \%$ & 2.137 & $5.2 \%$ & 2.113 & $4.0 \%$ & 2.157 & $6.2 \%$ & 2.115 \\
C7-C8 & 1.428 & 1.417 & $0.7 \%$ & 1.420 & $0.6 \%$ & 1.414 & $1.0 \%$ & 1.423 & $0.4 \%$ & 1.420 & $0.6 \%$ \\
Average relative error & $1.46 \%$ & - & $1.84 \%$ & - & $1.60 \%$ & - & $2.20 \%$ & - & $0.6 \%$ \\
\end{tabular}

${ }^{a}$ The experimental data is obtained from ref. 34 . 

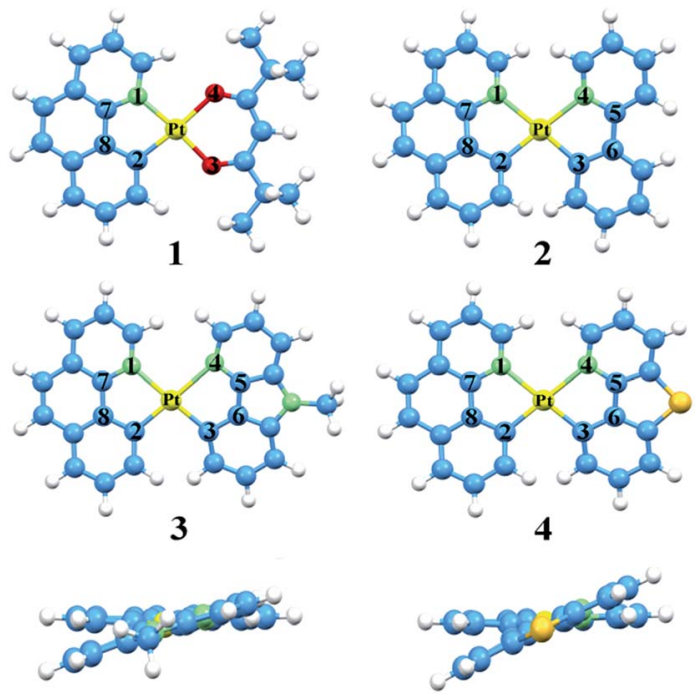

side view of 3

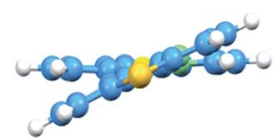

side view of 4
Fig. 2 Vertical view of the optimized geometries for 1-4 with the side view for 3 and 4 at the singlet ground state.

Pt-N. Meanwhile, the Pt-N3 and Pt-C4 are also consistent with the above property. At the $\mathrm{S}_{0}$ state, the Pt-N1 bond lengths of 24 are elongated by $0.125-0.165 \AA$ compared with complex 1 , which can be attributed to the trans effect in different ligands. ${ }^{55,56}$ In addition, compared with complexes 3-4, the bond lengths of $\mathrm{Pt}-\mathrm{C} 3$ and $\mathrm{Pt}-\mathrm{N} 4$ on the ppy ligand of 2 are shorter, while Pt-N1 and Pt-C2 bond lengths on the bzq ligand are elongated. These values reveal that the introduction of ppy ligand can increase its interaction with the metal atom and decrease the interaction between other ligand and metal atom. Obviously, the bond length of C5-C6 in 3 and 4 are shortened by 0.046-0.057 $\AA$, due to the substitution of ppy in the positions of both beta carbons by $\mathrm{N}$ and $\mathrm{S}$ atom to form Ncaz and Ndbt, which may strengthen the ligand internal-conjugation interaction. While, for $\mathrm{C} 7-\mathrm{C} 8$, the slight change within $0.008 \AA$ can be ignored. For the relevant bond angles of $\mathrm{Pt}$ and two ligands, except for the $\mathrm{C} 2-\mathrm{Pt}-\mathrm{N} 1$, the difference of others are induced by the five-member rings between $\mathrm{Pt}$ and ancillary ligands.

Since the $S_{0}$ and $T_{1}$ states participate in the process of phosphorescence, the geometry structures of $\mathrm{T}_{1}$ state worth noticing. The bond lengths of $\mathrm{Pt}-\mathrm{N} 1$ and $\mathrm{Pt}-\mathrm{C} 2$ for all complexes are shorter than the ground state. In other words, the bonds between metal and ligands are strengthened, which is likely to promote the charge transfer transition from metal centre to ligands. While the bond angles from $S_{0}$ to $T_{1}$ states have no distinct variation, especially for the designated complexes 2-4. The dihedral angle differences between $S_{0}$ and $\mathrm{T}_{1}$ states for all complexes are less than $2.0^{\circ}$. It is turned out that the complexes show better rigidity, which brings about a smaller nonradiative rate $\left(k_{\mathrm{nr}}\right){ }^{35}$

\subsection{Frontier molecular orbital properties}

In order to clearly reveal the electronic excitation of these $\mathrm{Pt}$ (II) complexes, it is essential to further investigate the frontier molecular orbital (FMOs), especially the highest occupied molecular orbital (HOMO) and lowest unoccupied molecular orbital (LUMO). The relevant orbital analysis along with the energy gaps at the $\mathrm{S}_{0}$ state are displayed in Fig. 3. The orbital compositions and distributions of selected FMOs are presented in Tables S2-S5 and Fig. S1. $\dagger$

For the electron density distributions of the HOMO, complex 1 is mainly located in $5 \mathrm{~d}(\mathrm{Pt})$ orbitals, the $\pi$ orbitals of bzq and dpm ligands. Obviously, the electron density distributions of HOMO for complexes 2 and $\mathbf{4}$ are rather similar, which are resided on the $5 \mathrm{~d}(\mathrm{Pt})$ orbitals and bzq ligand. Complex 3 is predominantly centralized on the $5 \mathrm{~d}(\mathrm{Pt})$ orbitals and the $\pi$ orbitals of Ncaz ligand. It is worth noting that the energy differences between HOMO and HOMO-1 of complexes 1-4 are $0.43(1), 0.10(2), 0.14$ (3) and $0.04 \mathrm{eV}(4)$ with the order of $4<2<$

Table 2 Selected main geometry parameters for 1-4 at the ground and lowest-lying triplet excited states

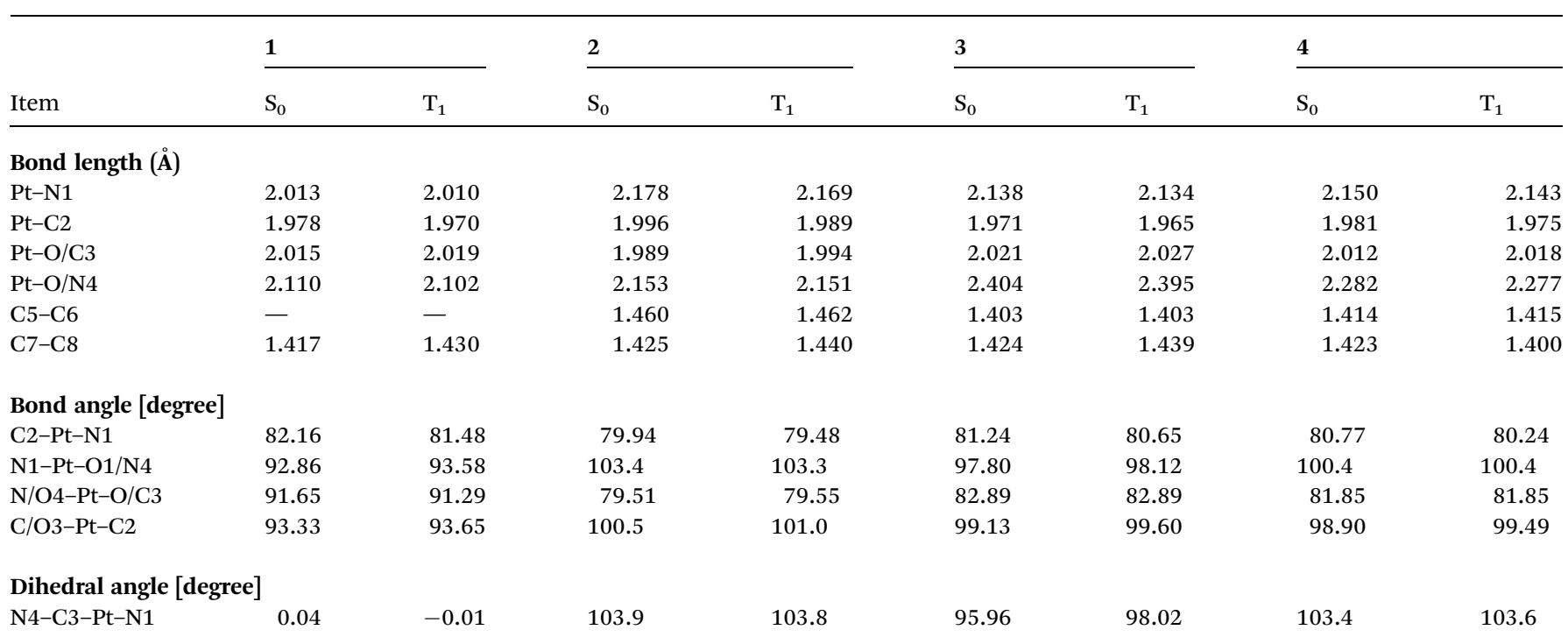




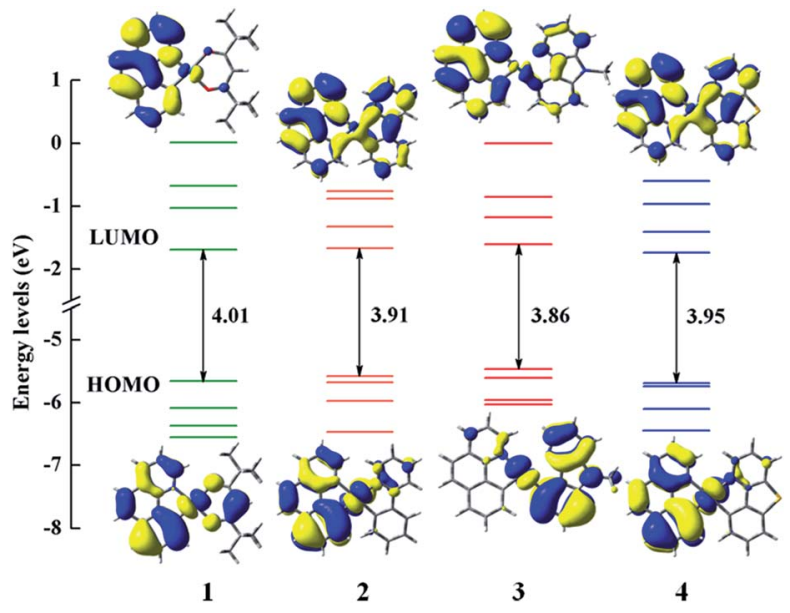

Fig. 3 Frontier molecular orbital energy levels, energy gaps (in eV) and orbital composition distributions of the HOMO and LUMO at the $\mathrm{S}_{0}$ state for 1-4.

$3<1$. It has been confirmed that the $H_{\text {SOC }}$ matrix element will be increased when the two highest occupied orbitals get close, which leads to a large radiative decay rate. ${ }^{14}$ Obviously, there are obvious differences in LUMO distributions of 2-4 compared with 1. The LUMO of 2 and 4 are delocalized on all ligands, while that in 1 is mainly centralized on the $\pi^{*}$ orbitals of bzq and a smaller contribution from $5 \mathrm{~d}$ orbitals of metal center. Combined with the diagram and data, metal $5 \mathrm{~d}(\mathrm{Pt})$ orbitals provide much contribution for the HOMO and the ligands constitute the majority of LUMO in all complexes, which demonstrates the transition nature from the HOMO to LUMO are metal-to-ligand transfer (MLCT) mixed with ligand-to-ligand (LLCT). As presented in the Fig. 3, the HOMO-LUMO gaps of complexes 2-4 are smaller owing to introducing the different ligands. So the transition from HOMO to LUMO is effective and the higher HOMO energy leads to a better ability of holeinjection compared with 1 . In addition, the LUMO energy level of 4 is calculated to be $-1.743 \mathrm{eV}$, which is lower than other complexes. The lower LUMO energy level could be caused by the better electron delocalization in the entire molecule to stabilize the unoccupied molecular orbitals. According to the calculated results, different ancillary ligands have significant influence on the distribution of FMOs, which may alter the energy gap and phosphorescence efficiency.

\subsection{Charge transportation properties}

It is known that low energy barrier for the injection and transport balance between the hole and electron of luminescent complexes are pivotal for the high performance OLEDs. In order to quantitatively discuss the charge injection properties, the ionization potential (IP) and electron affinity (EA) have been calculated by DFT method, which are closely related to the HOMO and LUMO, respectively. ${ }^{55}$ In addition, there are vertical $\left(\mathrm{IP}_{\mathrm{v}} / \mathrm{EA}_{\mathrm{v}}\right)$ and adiabatic $\left(\mathrm{IP}_{\mathrm{a}} / \mathrm{EA}_{\mathrm{a}}\right)$ two processes. For photoluminescent materials, a smaller IP value implies easier hole injection ability. On the other hand, a larger EA value facilitates the electrons injection capacity. The hole extraction potential (HEP) and electron extraction potential (EEP) are studied to evaluate the extraction potentials of the hole and electron.

According to the semiclassical Marcus theory, ${ }^{57}$ the intermolecular charge (hole and electron) transfer rate $\left(K_{\mathrm{et}}\right)$ can be expressed as follows:

$$
K_{\text {et }}=\left(\frac{4 \pi^{2}}{h}\right) \frac{V^{2}}{\sqrt{4 \pi \lambda K_{\mathrm{B}} T}} \exp \left(-\frac{\lambda}{4 K_{\mathrm{B}} T}\right)=A \exp \left(-\frac{\lambda}{4 K_{\mathrm{B}} T}\right)
$$

where $T$ and $K_{\mathrm{B}}$ are the temperature and Boltzmann constant, respectively. $\lambda$ represents the reorganization energy. $h$ is the Plank constant. $V$ is the electronic coupling terms for the adjacent molecules in the dimensional stacking, which is determined by the overlap of molecular orbitals. As shown in eqn (7), the $K_{\text {et }}$ is governed by two major factors that are $\lambda$ and $V$, respectively. Herein, taking the crystal structure of experimental complex 1 as example, the $V$ of hole and electron are calculated through employing ADF program package ${ }^{58}$ with PBE0/TZP method. From the calculation results, the $V$ of $\mathbf{1}$ for electron transfer is $0.0510 \mathrm{eV}$, and for hole transfer is $0.0156 \mathrm{eV}$, respectively. In comparison with previous work, it is negligibly small. ${ }^{59}$ Meanwhile, owing to the restricted intermolecular charge transfer range in solid state, the transfer rate of charge has been indicated to be mainly affected by reorganization energy $\lambda .^{60,61}$ From the eqn (7), a smaller $\lambda$ is the key factor for the faster charge transport property, which can be defined by the following relations:

$$
\begin{aligned}
& \lambda_{\mathrm{h}}=\lambda_{0}+\lambda_{+}=\mathrm{IP}_{\mathrm{v}}-\mathrm{HEP} \\
& \lambda_{\mathrm{e}}=\lambda_{0}+\lambda_{-}=\mathrm{EEP}-\mathrm{EA}_{\mathrm{v}}
\end{aligned}
$$

All the relevant calculation values of complexes 1-4 are shown in Table 3.

As can be seen in Table 3, complexes $\mathbf{2}$ and $\mathbf{3}$ have smaller IP values which are related to their higher HOMO energy levels than complex $\mathbf{1}$. Namely, the complexes $\mathbf{2}$ and $\mathbf{3}$ have better hole injection ability due to the introduction of ppy and Ncaz ligands. Meanwhile, the EA values of complexes $\mathbf{2}$ and $\mathbf{4}$ are larger than others, which can enhance electron injection ability and agree with their lower LUMO energy. From the Table 3, the reorganization energies of electron $\left(\lambda_{\mathrm{e}}\right)$ transport of all complexes are smaller than their hole $\left(\lambda_{h}\right)$ except for $\mathbf{1}$. Therefore, the complexes replaced the dpm with different ligands tend to be electron transporting materials. In addition, complex $\mathbf{3}$ is the promising bipolar transporting material because the difference between hole and electron reorganization values are

Table 3 lonization potentials (IPs), extraction potentials, electron affinity (EAs) and reorganization energy ( $\lambda$ ) of 1-4 (unit in eV)

\begin{tabular}{lllllllll}
\hline & IP(v) & IP( $(a)$ & HEP & EA(v) & EA(a) & EEP & $\lambda_{\text {hole }}$ & $\lambda_{\mathrm{e}}$ \\
\hline $\mathbf{1}$ & 6.85 & 6.77 & 6.68 & 0.29 & 0.42 & 0.56 & 0.17 & 0.27 \\
2 & 6.74 & 6.57 & 6.19 & 0.43 & 0.52 & 0.61 & 0.54 & 0.18 \\
3 & 6.62 & 6.51 & 6.39 & 0.32 & 0.41 & 0.50 & 0.23 & 0.18 \\
4 & 6.82 & 6.75 & 6.44 & 0.54 & 0.62 & 0.71 & 0.38 & 0.17
\end{tabular}


quite small $(0.05 \mathrm{eV})$, which is an important factor for the OLEDs devices. Generally speaking, the ancillary ligands can not only increase the abilities of hole and electron injection but also affect the balance of charge transfer.

\subsection{Phosphorescence emission properties}

On the basis of the optimized lowest triplet excited-state geometries, the phosphorescence properties of all the investigated complexes were calculated. The results including the lowest energy emission wavelengths and transition characters are shown in Table 4. The natural transition orbitals (NTOs) of complexes 14 are plotted in Fig. 4 and S2. $\dagger$ The unoccupied NTOs refer to the as "electron" transition orbitals while the occupied NTOs mean the "hole" transition orbitals. Furthermore, the orbital compositions distribution related to phosphorescence emission of complexes 1-4 are summarized in Table S6. $\dagger$

As we can know from the Table 4, the calculated emission wavelength is $537 \mathrm{~nm}$ for complex $\mathbf{1}$, which matches well with the experimental value of $505 \mathrm{~nm}$. The phosphorescence emissions of 2-4 at 535, 529, and $532 \mathrm{~nm}$ are slightly hypochromatic shifted (2$8 \mathrm{~nm}$ ) compared with that of $\mathbf{1}$, which reveals that the designed complexes 2-4 have a great trend to be bluish-green luminescent materials. Meanwhile, the emission wavelengths of complexes 1, 2 and $\mathbf{4}$ are nearly the same due to the similar electronic density distribution between HOMO and LUMO. Additionally, the $\mathrm{T}_{1}$ states of these complexes are originated largely from HOMO to LUMO. As shown in Fig. 4 and S2, $\dagger$ for complexes 1-4, the NTO hole is largely distributed in the metal Pt and bzq ligand, while the NTO electron is delocalized in the bzq ligand. Thus, the transition natures of the investigated complexes at $\mathrm{T}_{1}$ state are assigned as ${ }^{3} \mathrm{MLCT} /{ }^{3} \mathrm{LC}_{\mathrm{bzq}}$. In Table S6, $\uparrow$ the compositions of $5 \mathrm{~d}(\mathrm{Pt})$ orbitals have not much differences and be retained for these new complexes, and the LUMO of the studied complexes are all located in bzq ligand, indicating the bzq ligand is the main ligand in this system. For complexes 1, 2 and 4, the HOMO orbitals are centred on the $5 \mathrm{~d}(\mathrm{Pt})$ orbitals and bzq moieties. The transition of $\mathrm{H} \rightarrow \mathrm{L}$ is assigned as $\left[\mathrm{d}(\mathrm{Pt})+\pi(\mathrm{bzq}) \rightarrow \pi^{*}(\mathrm{bzq})\right]$ with ${ }^{3} \mathrm{MLCT} /{ }^{3} \mathrm{ILCT}$ (interligand charge transfer) transition characters. However, it can be found that the HOMO of complex 3 is localized on $5 \mathrm{~d}(\mathrm{Pt})$ orbitals, bzq and Ncaz ligands, it is described as $\left[\mathrm{d}(\mathrm{Pt})+\pi(\mathrm{bzq})+\pi\left(\mathrm{C}^{\wedge} \mathrm{N}\right) \rightarrow\right.$ $\left.\pi^{*}(\mathrm{bzq})\right]$ with ${ }^{3} \mathrm{MLCT} /{ }^{3} \mathrm{LLCT}$ transition characters.

Table 4 Calculated phosphorescent emissions of complexes 1-4 together with the experimental value

\begin{tabular}{|c|c|c|c|c|c|}
\hline & States & $\begin{array}{l}\lambda(\mathrm{nm}) / \\
E(\mathrm{eV})\end{array}$ & $\begin{array}{l}\lambda_{\text {expt }} \\
(\mathrm{nm})\end{array}$ & Main configuration & Assignment \\
\hline 1 & $\mathrm{~T}_{1}$ & $537 / 2.31$ & 505 & $\begin{array}{l}\mathrm{H} \rightarrow \mathrm{L}(72 \%) \\
\mathrm{H}-2 \rightarrow \mathrm{L}(14 \%)\end{array}$ & $\begin{array}{l}\text { MLCT/ILCT } \\
\text { MLCT/ILCT }\end{array}$ \\
\hline 2 & $\mathrm{~T}_{1}$ & $535 / 2.32$ & & $\begin{array}{l}\mathrm{H} \rightarrow \mathrm{L}(67 \%) \\
\mathrm{H} \rightarrow \mathrm{L}+1(10 \%)\end{array}$ & $\begin{array}{l}\text { MLCT/ILCT } \\
\text { MLCT/ILCT }\end{array}$ \\
\hline 3 & $\mathrm{~T}_{1}$ & $529 / 2.34$ & & $\begin{array}{l}\mathrm{H} \rightarrow \mathrm{L}(52 \%) \\
\mathrm{H}-1 \rightarrow \mathrm{L}(17 \%)\end{array}$ & $\begin{array}{l}\text { MLCT/LLCT } \\
\text { MLCT/LLCT }\end{array}$ \\
\hline 4 & $\mathrm{~T}_{1}$ & $532 / 2.33$ & & $\begin{array}{l}\mathrm{H} \rightarrow \mathrm{L}(65 \%) \\
\mathrm{H} \rightarrow \mathrm{L}+1(10 \%)\end{array}$ & $\begin{array}{l}\text { MLCT/ILCT } \\
\text { MLCT/LLCT }\end{array}$ \\
\hline
\end{tabular}
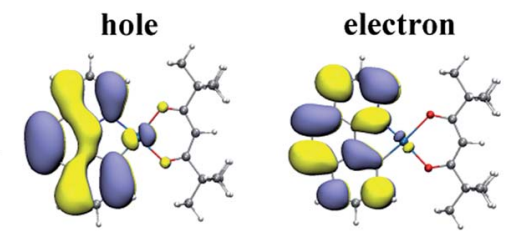

4
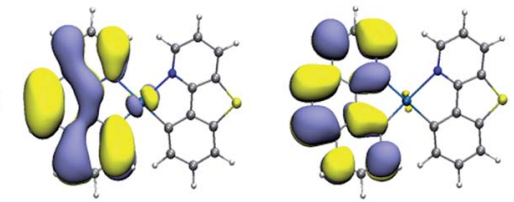

Fig. 4 NTO plots at the optimized $T_{1}$ states for 1 and 4

\subsection{Radiative decay rate}

Phosphorescence efficiency $\left(\Phi_{\mathrm{PL}}\right)$ of OLEDs is determined by the radiative decay rate constant $\left(k_{\mathrm{r}}\right)$ and non-radiative decay rate constant $\left(k_{\mathrm{nr}}\right)$, hence the theoretical analysis of $k_{\mathrm{r}}$ is imperative. Based on the optimized geometries of emissive states for all complexes, $k_{\mathrm{r}}$ and ZFS parameters are evaluated by TDDFT with the eqn (4) and (6). According to the eqn (4), the value of $k_{\mathrm{r}}$ is controlled by three factors including the SOC matrix elements between the $\mathrm{S}_{n}$ and $\mathrm{T}_{m}$ states, the oscillator strength $f_{n}$ of $\mathrm{S}_{n}$ and the energy gap among the coupled states. To ensure the effective coupling, only the first 10 singlet excited states and the lowlying triplet excited states $T_{m}$ which locate below the $S_{1}$ are taken into consideration. The calculated values of $k_{\mathrm{r}}$ and ZFS for all complexes are shown in Table 5.

Through the complete comparison, the $k_{\mathrm{r}}$ of complex 1 is in the same order of magnitude with the experimental value, indicating that the theoretical method used here is credible. The complexes 2-4 have sizable $k_{\mathrm{r}}$ compared with 1 implying that our design is reasonable for OLED materials. It is obvious that the replacement of ancillary ligands is beneficial to the radiative decay process, because the increased conjugation and rigidity of different ligands in cyclometalated Pt(II) complexes can lead to a large $k_{\mathrm{r}}$. In addition, the tendency of $\Delta E(\mathrm{ZFS})$ parameters are nearly consistent with the $k_{\mathrm{r}}$, except for 3 . The abnormal phenomenon of complex $\mathbf{3}$ is induced by the energy shifts of three near-equal substates.

In order to further analyze radiative decay process, the diagrams of involved $S_{n}$ and $T_{m}$ are collected in Fig. 5 and the relevant information is listed in Tables S7-S10. $\dagger$ The effective SOC can be achieved when ${ }^{1}$ MLCT and ${ }^{3}$ MLCT excitation configurations related to the $\mathrm{S}_{n}$ and $\mathrm{T}_{m}$ states possess the different occupied $5 \mathrm{~d}(\mathrm{Pt})$ orbitals and identical unoccupied $\pi^{*}$ orbitals. From the

Table 5 Calculated radiative decay rate constants $k_{\mathrm{r}}\left(10^{4} \mathrm{~s}^{-1}\right)$ and ZFS $\left(\mathrm{cm}^{-1}\right)$ of $1-4$ at the optimized $T_{1}$ geometries

\begin{tabular}{rrrrrll}
\hline & $k_{\mathrm{r}}^{z}$ & \multicolumn{1}{c}{$k_{\mathrm{r}}^{y}$} & \multicolumn{1}{c}{$k_{\mathrm{r}}^{x}$} & \multicolumn{1}{c}{$k_{\mathrm{r}}$} & $k_{\mathrm{r}, \exp }$ & $\Delta E(\mathrm{ZFS})$ \\
\hline $\mathbf{1}$ & 13.45 & 0.39 & 0.09 & 4.64 & 1.0 & 15.34 \\
$\mathbf{2}$ & 84.58 & 9.99 & 30.45 & 41.67 & - & 40.55 \\
$\mathbf{3}$ & 11.04 & 3.40 & 3.99 & 6.14 & - & 14.84 \\
$\mathbf{4}$ & 90.58 & 17.00 & 4.20 & 37.26 & - & 20.27
\end{tabular}




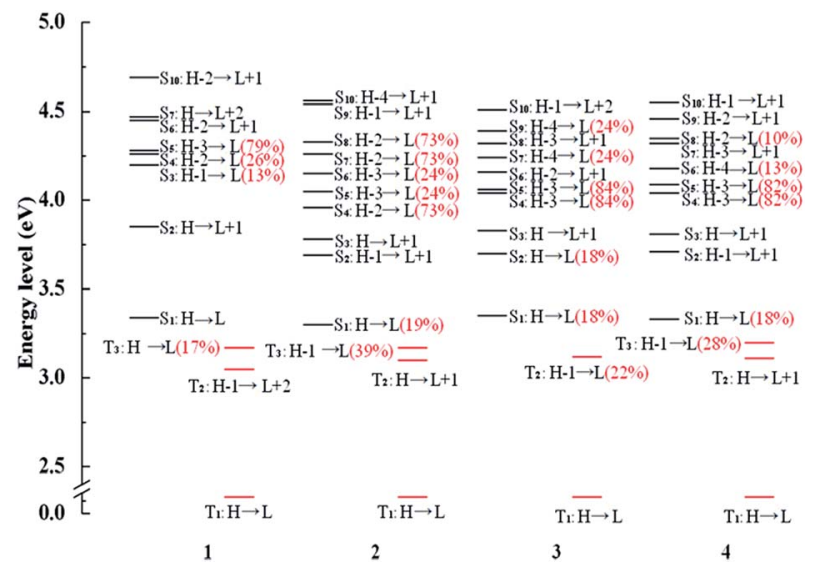

Fig. 5 Energy level diagrams of selected excited states between the triplet excited states $T_{m}$ and singlet excited states $S_{n}$ with the MLCT composition of major orbitals for complexes 1-4.

Fig. 5, the $\mathrm{T}_{1}$ state of complex 1 has three coupling $\mathrm{S}_{n}$ states, $\mathrm{S}_{3}, \mathrm{~S}_{4}$, and $S_{5}$ and three states $\left(S_{3}, S_{4}\right.$ and $\left.S_{5}\right)$ coupling with $T_{3}$ state. There are four states coupling with $\mathrm{T}_{1}$ state and six states coupling with $\mathrm{T}_{3}$ state in complex 3 . As for the complex 2, there are five utilizable singlet-excited states working on $\mathrm{T}_{1}$ state and six states can be coupled with $\mathrm{T}_{3}$ state. What is more, the intersystem crossing (ISC) from $S_{1}$ state to $T_{3}$ state is enhanced by the strong SOC process due to the transition from $T_{3}$ state to $S_{n}$ states is able to borrow intensity from the $\mathrm{S}_{1}$ state. ${ }^{62}$ Namely, the $\mathrm{H}-1$ to L transition of $\mathrm{T}_{3}$ state has the similar effect with the $\mathrm{H}$ to $\mathrm{L}$ transition. As a result, the $S_{1}$ state is allowed to coupling with $T_{3}$ state and the vertical excitation energy gap between the two coupled excited states is the closest, which also holds the same with 4 . Therefore, the newly designed complexes $\mathbf{2}$ and $\mathbf{4}$ have the larger radiative decay rate constants markedly.

\subsection{Nonradiative decay rate}

The nonradiative decay rate $\left(k_{\mathrm{nr}}\right)$ is the other factor to govern the $\Phi_{\mathrm{PL}}$. There are temperature-independent and temperaturedependent nonradiative decay processes. According to the energy gap law, the key to qualitative evaluation of the temperature-independent nonradiative decay rate are the energy gap between $T_{1}$ and $S_{0}$ states and the structural distortion that can be measured by Stokes shift. ${ }^{63}$ The more rigid the complex structure, the smaller the Stokes shift value. The corresponding values of Stokes shift are $0.84,0.66,0.68$ and $0.67 \mathrm{eV}$. It demonstrates that the introduction of different ligands is able to enhance the rigidity and reduce the structural distortion. The energy difference between $T_{1}$ and $S_{0}$ states is large and then the nonradiative decay pathway is less accessible. The calculated parameters of energy gaps for all complexes are 2.05, 2.03, 2.03 and $2.04 \mathrm{eV}$, respectively. As mentioned above, the temperatureindependent nonradiative decay rates of 2-4 are smaller than $\mathbf{1}$.

There is another significant process called temperaturedependent nonradiative decay pathway. Concretely speaking, the triplet excited state $\left({ }^{3} \mathrm{ES}\right)$ gets to short-lived metal-centred $\left({ }^{3} \mathrm{MC}\right)$ by the thermal population and then returns back to the

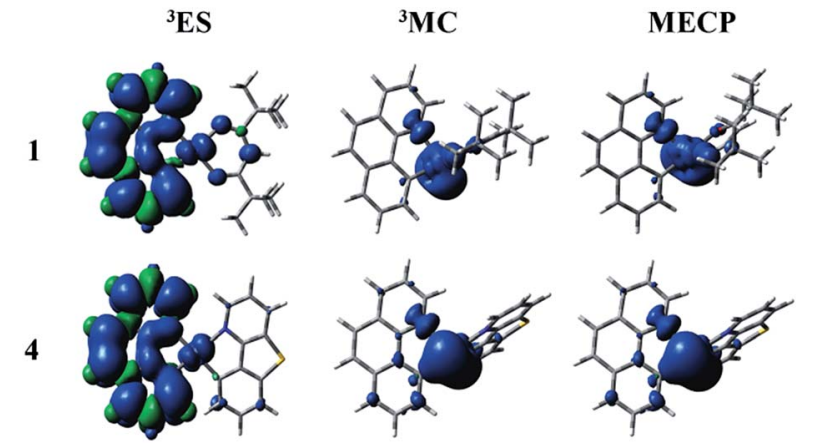

Fig. 6 The spin density of the lowest triplet excited state (left), ${ }^{3} \mathrm{MC}$ (middle) and MECP (right) states for complexes 1 and 4.

ground state $\left({ }^{1} \mathrm{GS}\right)$ through the minimum energy crossing point (MECP) in the relevant potential energy surfaces. From the previous studies, the temperature-dependent nonradiative decay rate constant will be decreased by the two factors: (1) a big activation barrier between the ${ }^{3} \mathrm{ES}$ and ${ }^{3} \mathrm{MC}$ states; (2) a higher relative energy of MECP. The MECP were constructed through the code developed by Harvey et al. ${ }^{64,65}$ The spin density plots of the studied complexes are depicted in Fig. 6 and S3. $\dagger$ The detailed description related to the spin density of complexes 1-4 for all ligands and metal Pt are listed in Table S11. $\dagger$ Besides, the intrinsic potential energy curves are shown in Fig. S4-S7. $\dagger$ At the lowest triplet excited state $\left({ }^{3} \mathrm{ES}\right)$, the spin density distributions of all complexes are mainly concentrated on the bzq ligand (93.85-95.50\%) and metal Pt (3.98-4.60\%). The electronic configurations of ${ }^{3} \mathrm{MC}$ states can be acquired by distorting the geometric structures. And the most distinctive feature of ${ }^{3} \mathrm{MC}$ states is that the spin density plots are mainly concentrated on the metal Pt. As shown in Fig. 6 and Table S11, $\dagger$ at the ${ }^{3} \mathrm{MC}$ states of complexes 1-4, there are about $65-75 \%$ contribution from the metal Pt. The distributions of MECP are similar with the ${ }^{3} \mathrm{MC}$ states, which are also centralized on the metal Pt. Unfortunately, the transition states (TS) between the ${ }^{3} \mathrm{ES}$ and ${ }^{3} \mathrm{MC}$ states have not been found out possibly due to the enhancement of molecular rigidity, so the schematics of the potential energy curves from ${ }^{3} \mathrm{ES}$ to ${ }^{3} \mathrm{MC}$ are smooth. The relative energies between the ${ }^{3} \mathrm{MC}$ and MECP are 4.24, 4.78, 4.86 and $4.43 \mathrm{kcal} \mathrm{mol}^{-1}$, respectively. There are no obvious differences of the energy gaps for all complexes, which indicate the temperature-dependent nonradiative decay rates of complexes 1-4 will not have too much difference.

\section{Conclusions}

The geometric structures, charge transfer performance, electronic and photophysical properties of four cyclometalated platinum(II) complexes 1-4 are systematically investigated by employing DFT and TDDFT methods. It should be noted that the bond lengths and angles of complexes 2-4 with different ligands instead of dpm ligand have no obvious differences from $S_{0}$ to $T_{1}$ state. Thus, complexes $\mathbf{2 - 4}$ have better molecular rigidity which can decrease the nonradiative decay rate. The newly designed complex $\mathbf{3}$ has balanced charge carrier 
injection/transport performance which is important for OLED materials. As for the phosphorescence properties, the ${ }^{3} \mathrm{MLCT} /{ }^{3} \mathrm{LLCT}$ character is assigned for 3 , while complexes $\mathbf{1}, 2$ and 4 are assigned as ${ }^{3} \mathrm{MLCT} /{ }^{3} \mathrm{ILCT}$. The radiative decay rate constants are presented in the following trend: $\mathbf{2}>\mathbf{4}>\mathbf{3}>\mathbf{1}$. Hence, it is inferred that complexes $\mathbf{2}$ and $\mathbf{4}$ may be excellent phosphorescent materials for the higher phosphorescence quantum efficiency. Combined with the above analysis, we come to the conclusion that reasonable modification and variation of the ancillary ligand will be beneficial to improve the phosphorescence quantum efficiency of complexes for the highly efficient OLED emitting materials.

\section{Acknowledgements}

This work was supported by the State Key Development Program for Basic Research of China (Grant No. 2013CB834801) and the Natural Science Foundation of China (Grant No. 21573088) and Young Scholar Training Program of Jilin University and Open Project Founding of Beijing National Laboratory for Molecular Sciences (BNLMS).

\section{Notes and references}

1 L.-H. Xu, Q.-D. Ou, Y.-Q. Li, Y.-B. Zhang, X.-D. Zhao, H.-Y. Xiang, J.-D. Chen, L. Zhou, S.-T. Lee and J.-X. Tang, ACS Nano, 2016, 10, 1625-1632.

2 C.-W. Hsu, K. T. Ly, W.-K. Lee, C.-C. Wu, L.-C. Wu, J.-J. Lee, T.-C. Lin, S.-H. Liu, P.-T. Chou, G.-H. Lee and Y. Chi, ACS Appl. Mater. Interfaces, 2016, 8, 33888-33898.

3 R. Gomez-Bombarelli, J. Aguilera-Iparraguirre, T. D. Hirzel, D. Duvenaud, D. Maclaurin, M. A. Blood-Forsythe, H. S. Chae, M. Einzinger, T. Wu, G. Markopoulos, S. Jeon, H. Kang, H. Miyazaki, M. Numata, S. Kim, W. Huang, S. I. Hong, M. Baldo, R. P. Adams and A. Aspuru-Guzik, Nat. Mater., 2016, 15, 1120-1127.

4 Y. Wu, G.-G. Shan, H.-B. Li, S.-X. Wu, X.-Y. Ren, Y. Geng and Z.-M. Su, Phys. Chem. Chem. Phys., 2015, 17, 2438-2446.

5 L. Xiao, Z. Chen, B. Qu, J. Luo, S. Kong, Q. Gong and J. Kido, Adv. Mater., 2011, 23, 926-952.

6 S. Reineke, F. Lindner, G. Schwartz, N. Seidler, K. Walzer, B. Lussem and K. Leo, Nature, 2009, 459, 234-238.

7 L.-M. Xie, F.-Q. Bai, W. Li, Z.-X. Zhang and H.-X. Zhang, Phys. Chem. Chem. Phys., 2015, 17, 10014-10021.

8 F.-Q. Bai, J. Wang, B.-H. Xia, Q.-J. Pan and H.-X. Zhang, Dalton Trans., 2012, 41, 8441-8446.

9 K. O. Kirlikovali, J. C. Axtell, A. Gonzalez, A. C. Phung, S. I. Khan and A. M. Spokoyny, Chem. Sci., 2016, 7, 51325138.

10 A.-H. Liang, F.-Q. Bai, J. Wang, J.-B. Ma and H.-X. Zhang, Aust. J. Chem., 2014, 67, 1522-1531.

11 B.-Q. Liu, L. Wang, D.-Y. Gao, J.-H. Zou, H.-L. Ning, J.-B. Peng and Y. Cao, Light: Sci. Appl., 2016, 5, e16137.

12 A. Tronnier, S. Metz, G. Wagenblast, I. Muenster and T. Strassner, Dalton Trans., 2014, 43, 3297-3305.

13 P. Pinter, H. Mangold, I. Stengel, I. Munster and T. Strassner, Organometallics, 2016, 35, 673-680.
14 A. F. Rausch, L. Murphy, J. A. G. Williams and H. Yersin, Inorg. Chem., 2012, 51, 312-319.

15 F.-Q. Bai, X. Zhou, B.-H. Xia, T. Liu, J.-P. Zhang and H.-X. Zhang, J. Organomet. Chem., 2009, 694, 1848-1860.

16 X.-Q. Zhang, Y.-M. Xie, Y.-H. Zheng, F. Liang, B. Wang, J. Fan and L.-S. Liao, Org. Electron., 2016, 32, 120-125.

17 B. Yang, S. Huang, J. Zhong and H. Zhang, Org. Electron., 2015, 19, 7-14.

18 J.-H. Du, Y.-F. Luo, X.-H. Xie, W.-H. Hu and W. Shen, Mol. Simul., 2016, 42, 1035-1041.

19 S.-Y. Chang, Y.-M. Cheng, Y. Chi, Y. C. Lin, C.-M. Jiang, G.-H. Lee and P.-T. Chou, Dalton Trans., 2008, 48, 6901-6911.

20 Y. Chi and P.-T. Chou, Chem. Soc. Rev., 2010, 39, 638-655.

21 S.-B. Ko, J.-S. Lu, Y. Kang and S. Wang, Organometallics, 2013, 32, 599-608.

22 F. M. Hwang, H.-Y. Chen, P.-S. Chen, C.-S. Liu, Y. Chi, C.-F. Shu, F.-L. Wu, P.-T. Chou, S.-M. Peng and G.-H. Lee, Inorg. Chem., 2005, 44, 1344-1353.

23 T. Tsuzuki, N. Shirasawa, T. Suzuki and S. Tokito, Adv. Mater., 2003, 15, 1455-1458.

24 X.-N. Li, Z.-J. Wu, Z.-J. Si, H.-J. Zhang, L. Zhou and X.-J. Liu, Inorg. Chem., 2009, 48, 7740-7749.

25 Y. C. Chiu, Y. Chi, J.-Y. Hung, Y.-M. Cheng, Y.-C. Yu, M.-W. Chung, G.-H. Lee, P.-T. Chou, C.-C. Chen, C.-C. Wu and H. Y. Hsieh, ACS Appl. Mater. Interfaces, 2009, 1, 433442.

26 C.-H. Yang, Y.-M. Cheng, Y. Chi, C.-J. Hsu, F.-C. Fang, K.-T. Wong, P.-T. Chou, C.-H. Chang, M.-H. Tsai and C.-C. Wu, Angew. Chem., Int. Ed., 2007, 46, 2418-2421.

27 S. Haneder, E. Da Como, J. Feldmann, J. M. Lupton, C. Lennartz, P. Erk, E. Fuchs, O. Molt, I. Munster, C. Schildknecht and G. Wagenblast, Adv. Mater., 2008, 20, 3325-3330.

28 Y. Unger, D. Meyer, O. Molt, C. Schildknecht, I. Münster, G. Wagenblast and T. Strassner, Angew. Chem., Int. Ed., 2010, 122, 10412-10414.

29 J. C. H. Chan, W. H. Lam, H.-L. Wong, N.-Y. Zhu, W.-T. Wong and V. W. W. Yam, J. Am. Chem. Soc., 2011, 133, 1269012705.

30 W. Wu, W. Wu, S. Ji, H. Guo, P. Song, K. Han, L. Chi, J. Shao and J. Zhao, J. Mater. Chem., 2010, 20, 9775-9786.

31 J. A. G. Williams, S. Develay, D. L. Rochester and L. Murphy, Coord. Chem. Rev., 2008, 252, 2596-2611.

32 M.-C. Tang, A. K.-W. Chan, M.-Y. Chan and V. W.-W. Yam, Top. Curr. Chem., 2016, 374, 46.

33 X. C. Hang, T. Fleetham, E. Turner, J. Brooks and J. Li, Angew. Chem., Int. Ed., 2013, 52, 6753-6756.

34 A. Bossi, A. F. Rausch, M. J. Leitl, R. Czerwieniec, M. T. Whited, P. I. Djurovich, H. Yersin and M. E. Thompson, Inorg. Chem., 2013, 52, 12403-12415.

35 W. H. Lam, E. S. H. Lam and V. W. W. Yam, J. Am. Chem. Soc., 2013, 135, 15135-15143.

36 Y.-F. Luo, Y.-Y. Xu, W.-T. Zhang, M. Li, R.-X. He and W. Shen, ChemPhysChem, 2016, 17, 69-77.

37 J. S. Wilson, N. Chawdhury, M. R. A. Al-Mandhary, M. Younus, M. S. Khan, P. R. Raithby, A. Kohler and R. H. Friend, J. Am. Chem. Soc., 2001, 123, 9412-9417. 
38 Y. Kataoka, Y. Kitagawa, T. Kawakami and M. Okumura, J. Organomet. Chem., 2013, 743, 163-169.

39 Z. A. Siddique, Y. Yamamoto, T. Ohno and K. Nozaki, Inorg. Chem., 2003, 42, 6366-6378.

40 S. Obara, M. Itabashi, F. Okuda, S. Tamaki, Y. Tanabe, Y. Ishii, K. Nozaki and M.-a. Haga, Inorg. Chem., 2006, 45, 8907-8921.

41 S. Lee, S. O. Kim, H. Shin, H. J. Yun, K. Yang, S. K. Kwon, J. J. Kim and Y. H. Kim, J. Am. Chem. Soc., 2013, 135, 14321-14328.

42 J. M. Younker and K. D. Dobbs, J. Phys. Chem. C, 2013, 117, 25714-25723.

43 H. Yersin, W. Humbs and J. Strasser, Coord. Chem. Rev., 1997, 159, 325-358.

$44 \mathrm{H}$. Yersin, W. Humbs and J. Strasser, Electronic and Vibronic Spectra of Transition Metal Complexes II, Springer, Berlin Heidelberg, 1997, vol. 191, pp. 153-249.

45 M. J. Frisch, G. W. Trucks, H. B. Schlegel, G. E. Scuseria, M. A. Robb, J. R. Cheeseman, G. Scalmani, V. Barone, B. Mennucci, G. A. Petersson, H. Nakatsuji, M. Caricato, X. Li, H. P. Hratchian, A. F. Izmaylov, J. Bloino, G. Zheng, J. L. Sonnenberg, M. Hada, M. Ehara, K. Toyota, R. Fukuda, J. Hasegawa, M. Ishida, T. Nakajima, Y. Honda, O. Kitao, H. Nakai, T. Vreven, J. A. Montgomery Jr, J. E. Peralta, F. Ogliaro, J. M. Bearpark, J. Heyd, E. Brothers, K. N. Kudin, V. N. Staroverov, R. Kobayashi, J. Normand, K. Raghavachari, A. Rendell, J. C. Burant, S. S. Iyengar, J. Tomasi, M. Cossi, N. Rega, J. M. Millam, M. Klene, J. E. Knox, J. B. Cross, V. Bakken, C. Adamo, J. Jaramillo, R. Gomperts, R. E. Stratmann, O. Yazyev, A. J. Austin, R. Cammi, C. Pomelli, J. W. Ochterski, R. L. Martin, K. Morokuma, V. G. Zakrzewski, G. A. Voth, P. Salvador, J. J. Dannenberg, S. Dapprich, A. D. Daniels, Ö. Farkas, J. B. Foresman, J. V. Ortiz, J. Cioslowski and D. J. Fox, Gaussian 09 Revision D.01, Gaussian, Inc., Wallingford, CT, 2009.

46 V. Barone, M. Cossi and J. Tomasi, J. Chem. Phys., 1997, 107, 3210-3221.
47 M. Cossi, G. Scalmani, N. Rega and V. Barone, J. Chem. Phys., 2002, 117, 43-54.

48 P. J. Hay and W. R. Wadt, J. Chem. Phys., 1985, 82, 299-310. 49 C. Lee, W. Yang and R. G. Parr, Phys. Rev. B: Condens. Matter Mater. Phys., 1988, 37, 785-789.

50 J. P. Perdew, Phys. Rev. B: Condens. Matter Mater. Phys., 1986, 33, 8822-8824.

51 C. Adamo and V. Barone, J. Chem. Phys., 1999, 110, 61586170.

52 Y. Zhao and D. G. Truhlar, Theor. Chem. Acc., 2008, 120, 215241.

53 T. Yanai, D. P. Tew and N. C. Handy, Chem. Phys. Lett., 2004, 393, 51-57.

54 D. Jacquemin, E. A. Perpete, I. Ciofini and C. Adamo, J. Chem. Theory Comput., 2010, 6, 1532-1537.

55 T.-T. Feng, F.-Q. Bai, L.-M. Xie, Y. Tang and H.-X. Zhang, RSC Adv. , 2016, 6, 11648-11656.

56 M. K. Nazeeruddin, R. Humphry-Baker, D. Berner, S. Rivier, L. Zuppiroli and M. Graetzel, J. Am. Chem. Soc., 2003, 125, 8790-8797.

57 R. A. Marcus, J. Chem. Phys., 1956, 24, 966-978.

58 G. te Velde, F. M. Bickelhaupt, E. J. Baerends, C. F. Guerra, S. J. A. Van Gisbergen, J. G. Snijders and T. Ziegler, J. Comput. Chem., 2001, 22, 931-967.

59 X.-L. Ding, L.-Y. Zou, M.-S. Ma, H.-X. Zhang, Y.-X. Cheng and A.-M. Ren, Org. Electron., 2016, 31, 111-119.

60 B.-C. Lin, C.-P. Cheng and Z. P. M. Lao, J. Phys. Chem. A, 2003, 107, 5241-5251.

61 M. Malagoli and J. L. Bredas, Chem. Phys. Lett., 2000, 327, 1317.

62 E. Jansson, B. Minaev, S. Schrader and H. Agren, Chem. Phys., 2007, 333, 157-167.

63 N. I. Nijegorodov and W. S. Downey, J. Phys. Chem., 1994, 98, 5639-5643.

64 J. N. Harvey and M. Aschi, Phys. Chem. Chem. Phys., 1999, 1, 5555-5563.

65 J. N. Harvey, M. Aschi, H. Schwarz and W. Koch, Theor. Chem. Acc., 1998, 99, 95-99. 